\title{
ON THE RESOLUTION OF A PARABOLIC EQUATION IN A NONREGULAR DOMAIN OF $\mathbb{R}^{3}$
}

\author{
A. Kheloufi, R. LABbas and B.-K. SADAllah
}

\begin{abstract}
In this work we give new results of existence, uniqueness and maximal regularity of a solution to a parabolic equation set in a nonregular domain $Q$ with Cauchy-Dirichlet boundary conditions, where $\left.Q=\left\{\left(t, x_{1}\right) \in \mathbb{R}^{2}: 0<t<T ; \varphi_{1}(t)<x_{1}<\varphi_{2}(t)\right\} \times\right] 0, b\left[\subseteq \mathbb{R}^{3}\right.$ with some assumptions on the functions $\left(\varphi_{i}\right)_{i=1,2}$. The right-hand side term of the equation is taken in $L^{2}(Q)$. The method used is based on the approximation of the domain $Q$ by a sequence of subdomains $\left(Q_{n}\right)_{n}$ which can be transformed into regular domains. This work is an extension of the one space variable case studied in [12].
\end{abstract}

Mathematics subject classification (2010): 35K05, 35K20.

Keywords and phrases: parabolic equation, nonregular domains, anisotropic Sobolev space.

\section{REFERENCES}

[1] Yu. A. Alkhutov, $L_{P}$ - estimates of solutions of the Dirichlet problem for the heat equation in a ball, Journal of Mathematical Sciences, 142, 3 (2007), 2021-2032.

[2] V. N. Aref'Ev, L. A. BAgirov, Solutions of the heat equation in domains with singularities, Mathematical Notes, 64, 2 (1998), 139-153.

[3] E. A. BADERKO, On the solution of the boundary value problems for parabolic equations of high order in domains with curvilinear lateral boundaries, Diff, Urav., 12, 10 (1976), 1781-1792.

[4] V. Besov, The continuation of function in $L_{p}^{1}$ and $W_{p}^{1}$, Proc. Steklov Inst. Math., 89 (1967), 5-17.

[5] P. Grisvard, Elliptic Problems in Nonsmooth Domains, Monographs and Studies in Mathematics 24, Pitman, Boston, 1985.

[6] S. Hofmann, J. L. Lewis, The $L^{p}$ regularity problems for the heat equation in non-cylindrical domains, Journal of Functional Analysis, 220 (2005), 1-54.

[7] R. Labbas, A. Medeghri, B.-K. Sadallah, On a parabolic equation in a triangular domain, Applied Mathematics and Computation, 130 (2002), 511-523.

[8] R. Labbas, A. Medeghri, B.-K. Sadallah, An $L^{p}$ approach for the study of degenerate parabolic equation, EJDE, 2005, 36 (2005), 1-20.

[9] LadyZhensKaya-Solonnikov-URAL'TSEVA, Linear and Quasi-Linear Equations of Parabolic Type. A.M.S., providence, Rhode Island, 1968.

[10] J. L. Lions, E. Magenes, Problèmes aux Limites Non Homogènes et Applications, 1,2, Dunod, Paris, 1968.

[11] J. NeČAs, Les Méthodes Directes en Théorie des Equations Elliptiques, Masson, Paris, 1967.

[12] B.-K. Sadallah, Etude d'un Problème 2m-parabolique dans des domaines plan non rectangulaires, Boll. Un. Mat. Ital., 5, 2-B (1983), 51-112.

[13] G. SAVARÉ, Parabolic problems with mixed variable lateral conditions: an abstract approach, J. Math. Pures Appl., 76, (1997), 321-351. 